\title{
Low Cost Instrumentation for Electrical Impedance Tomography in Biomedical Applications
}

\author{
Hancong Wu, Jiabin Jia and Stewart Smith \\ School of Engineering, The University of Edinburgh
}

\section{Introduction}

Impedance measurements in biological and biomedical applications have been studied since the middle of the 20th century, pioneered by researchers such as Nyboer [I]. One of the the earliest medical applications was the measurement of blood flow by impedance plethysmography, [I] while many people will be familiar with the measurement of body fat content via impedance electrodes found on bathroom scales. More recently, impedance measurement has been applied in cell biology through techniques such as electrical cell-substrate impedance sensing (ECIS) [2], and electrical impedance tomography (EIT) [3]. Instrumentation for these techniques is commercially available but the high cost represents a barrier for widespread application in biosensing, especially for use in the developing world. Simic [4] has pioneered the application of the AD5933 impedance analyser for low-cost biosensing applications. The current work extends this by adding additional external circuitry based on [5] to allow the four-terminal impedance measurements required for EIT.

\section{Electrical Impedance Tomography}

Electrical Impedance Tomography (EIT) provides images of the conductivity distribution inside the material under test. In EIT an excitation current is forced between an adjacent pair of peripheral electrodes, while the induced potential difference is simultaneously acquired at the other electrode pairs. Excitation and acquisition are continuously and rapidly repeated until all the possible electrode combinations are measured. The cross-sectional image is then reconstructed to display the spatial distribution of electrical impedance.

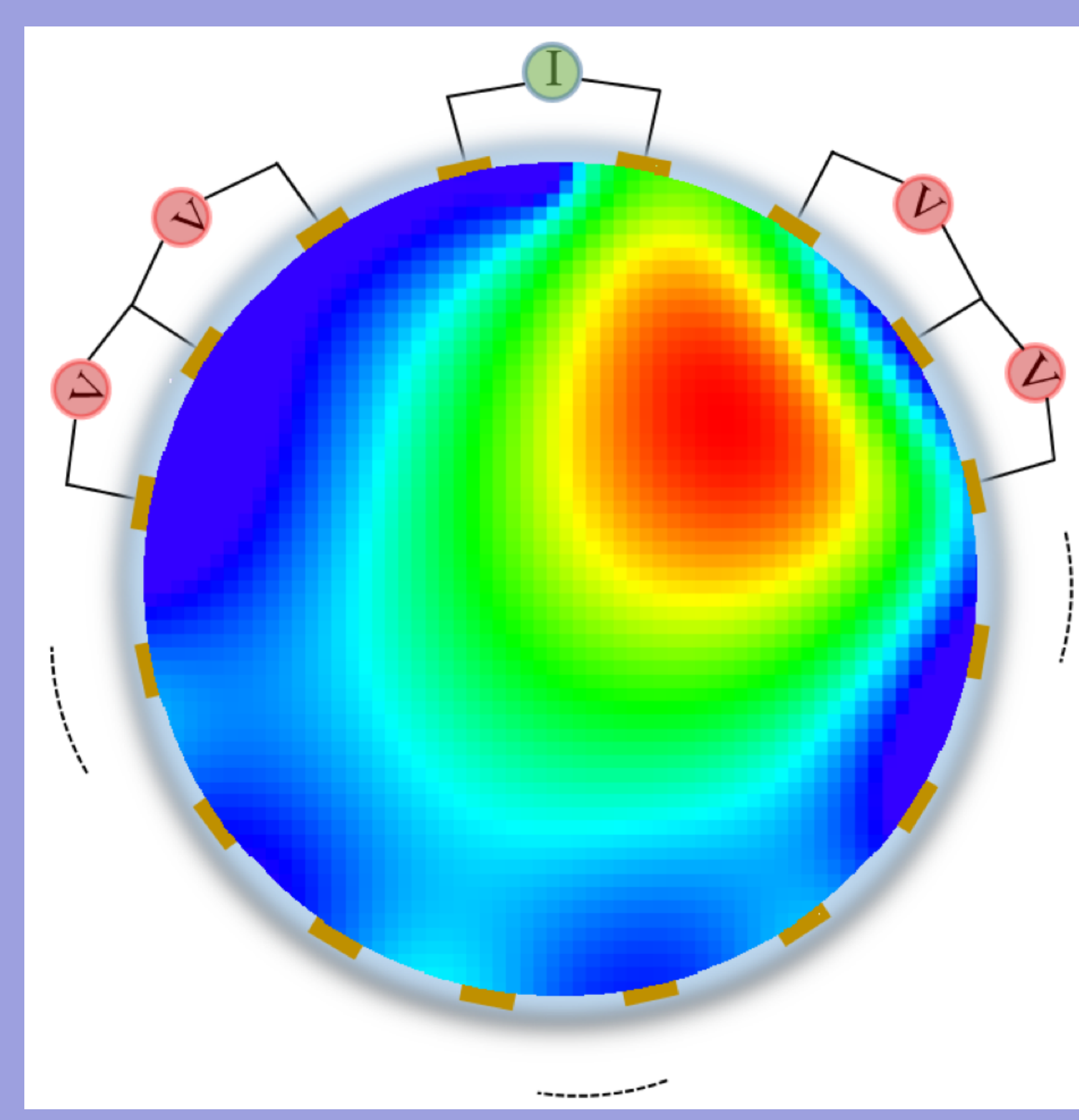

Typical applications of EIT include earth science and industrial process monitoring. As a low-cost and fast sensing technique, EIT has been used in research for pathological diagnosis in lung, breast and brain. Recently, imaging of biological cells has become a new application of EIT [6]. Due to the presence of the capacitive cell membrane, the electric field of EIT penetrates either the intracellular or extracellular domain at different frequencies. Multi-frequency EIT has the potential to allow new, non-invasive methods of characterising cells deep within tissue where traditional microscopy will fail.

\section{Circuit Design}

The external circuit is designed to apply the required excitation current and allow measurement of the resulting potential. It consists of four modules: an isolation filter, the excitation current source, a differential voltage sensing stage and a feedback current source.

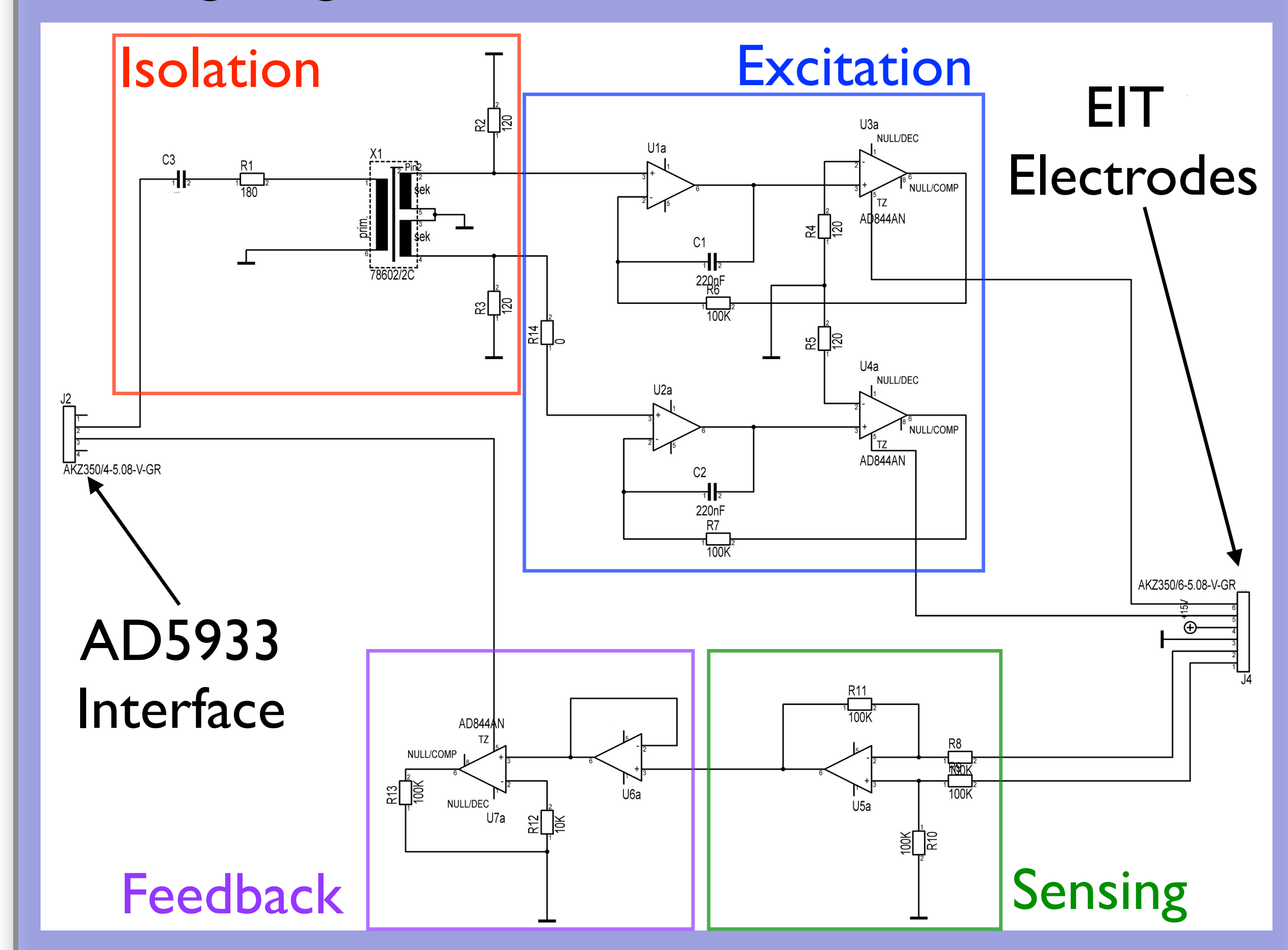

The AD5933 impedance analyser applies a voltage across the material under test (MUT) and measures the current in a two-terminal measurement. The first stage (isolation) in the external circuit uses a $1: 1: 1$ transformer to turn the AD5933 voltage output into a pair of complementary signals, it also removes any DC components and isolates the external measurement circuit. The excitation block is a pair of voltage controlled current sources (VCCS) that supply complementary AC currents to the EIT electrodes. The resulting potential is sensed with a differential amplifier and the output voltage is transformed to a proportional current by a second VCCS in the feed back block. The VCCS output is connected to the AD5933 current sensing terminal.

\section{Conclusions and Next Steps}

These results show that this low cost, complex impedance measurement system is capable of the measurements required for EIT image reconstruction. However, there are still issues with the accuracy and resolution of the measurements that require further work. The next steps in this research activity will include:

- Design of a switching control system which will allow automation of the EIT measurements and generation of data files for use in the EIDORS reconstruction software. This will also enable other forms of EIT measurement, such as frequency dependent imaging.

- Improve the analogue side of the measurement system, for example to remove the need for a transformer in the isolation stage and a second VCCS in the feedback stage.

- Develop software to control the measurement hardware and EIDORS, in order to create an integrated EIT measurement system for future biomedical applications.

\section{Measurements and Results}

A tissue culture dish model consisting of a ring of 16 EIT electrodes surrounding a liquid reservoir has been constructed. This is filled with a conductive solution of saline. A piece of rubber eraser is used to represent a low conductivity volume while a piece of cucumber soaked in saline will represent biological tissue. The measurement setup is shown below. Note that in this initial experiment the switching of signals to make the 208 measurements is performed manually through the protoboard.

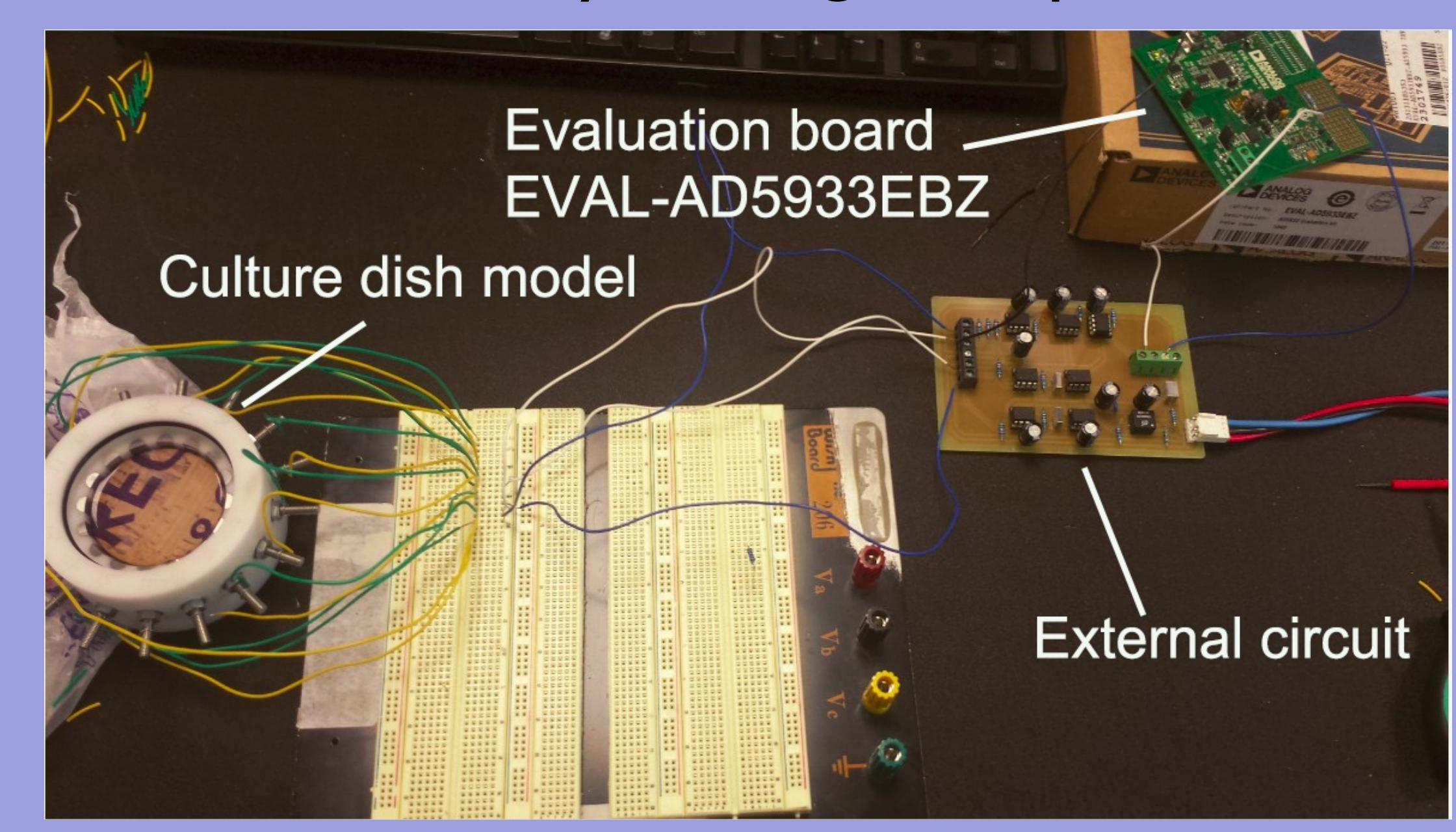

The impedence measurements from the AD5933 are used with the EIDORS [7] open source EIT image reconstruction software to obtain the image shown below.

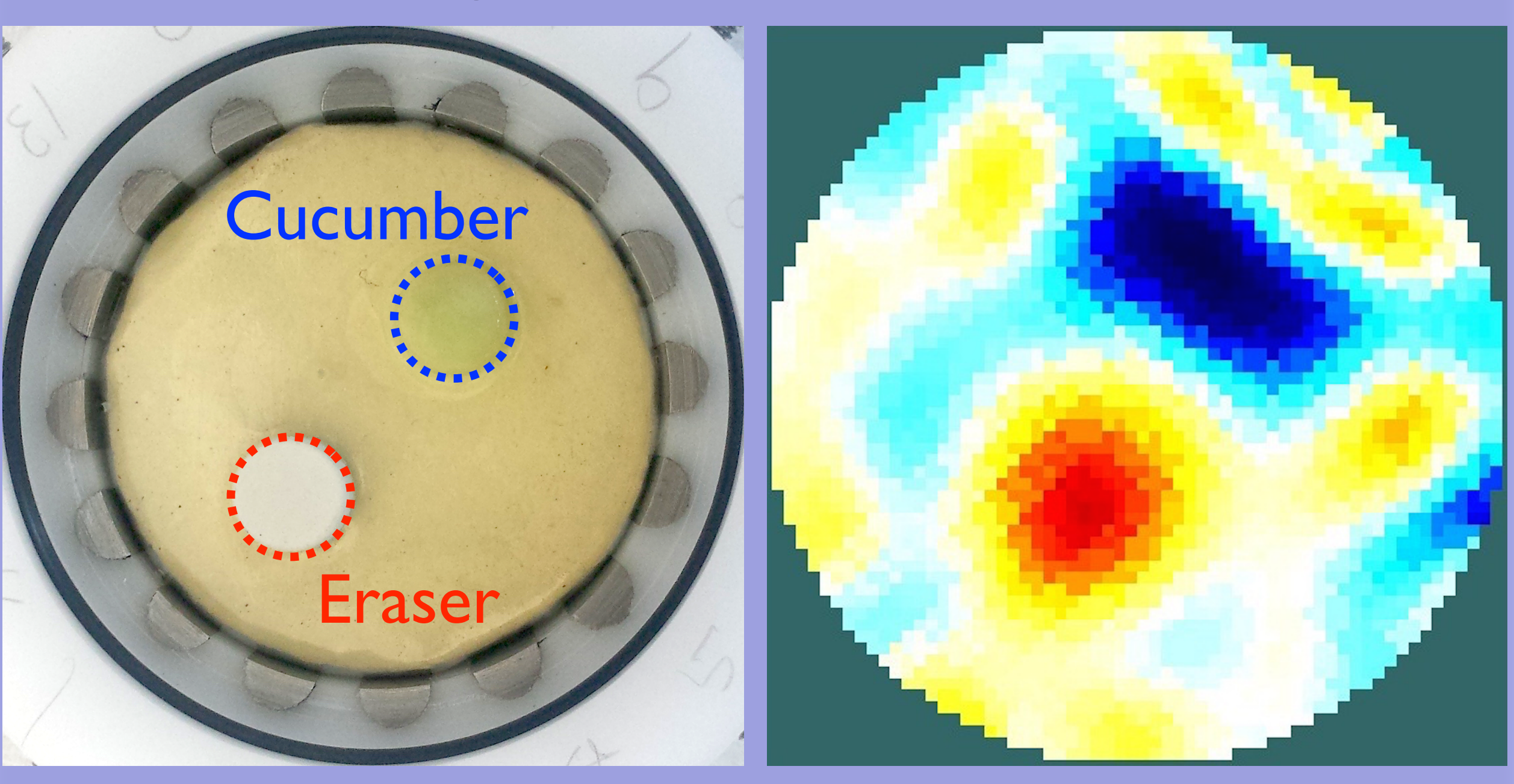

In the EIT image, areas of high impedance are red, while areas of low impedance are blue. While the results clearly show the eraser as an area of high impedance as expected, the low impedance represented by the cucumber requires more explanation.

The cucumber piece is thicker than the depth of the saline solution so if it is assumed to have a similar conductivity then the effective impedance may be lower.

The apparent spread in area of the low impedance region compared to the size of the cucumber piece may be due to it moving during the measurement. This can take a considerable time due to manual switching of the electrical connections between the electrodes and the the impedance measurement system.

\section{References}

[I]J. Nyboer, et al., Circulation, vol. 2, no. 6, pp. 81 I-82I, Dec. 1950.

[2] C. R. Keese and I. Giaever, IEEE Eng. Med. Biol. Mag., vol. I3, no. 3, pp. 402-408, 1994

[3] B. H. Brown, J. Med. Eng. Technol., vol. 27, no. 3, pp. 97-108, Jan. 2003.

[4] M. Simic, 2 Ist Telecommunications Forum (TELFOR), 2013, pp. 573-576, 2013.

[5] C. Margo, et al., Physiological Measurement, vol. 34, no. 4, pp. 39l-405, Apr. 2013.

[6] T. Sun, et al., Biosensors and Bioelectronics, vol. 25, no. 5, pP. I 109-III5, Jan. 2010.

[7] A.Adler and W.R.B. Lionheart, Physiological Measurement, vol. 27, no. 5, S25-S42, Apr. 2006. 